\title{
Health economic studies of colorectal cancer and the contribution of administrative data: A systematic review
}

\author{
Elizabeth Lemmon $^{1} \odot \mid$ Catherine R. Hanna ${ }^{2} \odot \mid$ Peter Hall $^{3} \odot \mid$ Eva J. A. Morris ${ }^{4} \odot$
}

${ }^{1}$ Edinburgh Health Economics, University of Edinburgh, Edinburgh, UK

${ }^{2}$ CRUK Clinical Trials Unit, Institute of Cancer Sciences, University of Glasgow, Glasgow, UK

${ }^{3}$ Edinburgh Cancer Research Centre, University of Edinburgh, Edinburgh, UK

${ }^{4}$ Nuffield Department of Population Health, University of Oxford, Oxford, UK

\section{Correspondence}

Elizabeth Lemmon, Edinburgh Clinical Trials Unit, Edinburgh Health Economics, University of Edinburgh, NINE BioQuarter, Edinburgh EH16 4UX, UK.

Email: elizabeth.lemmon@ed.ac.uk

Funding information

This study was supported by Cancer Research UK (C23434/A23706)

\begin{abstract}
Introduction: Several forces are contributing to an increase in the number of people living with and surviving colorectal cancer (CRC). However, due to the lack of available data, little is known about the implications of these forces. In recent years, the use of administrative records to inform research has been increasing. The aim of this paper is to investigate the potential contribution that administrative data could have on the health economic research of CRC.

Methods: To achieve this aim, we conducted a systematic review of the health economic CRC literature published in the United Kingdom and Europe within the last decade (2009-2019).

Results: Thirty-seven relevant studies were identified and divided into economic evaluations, cost of illness studies and cost consequence analyses. Conclusions: The use of administrative data, including cancer registry, screening and hospital records, within the health economic research of CRC is commonplace. However, we found that this data often come from regional databases, which reduces the generalisability of results. Further, administrative data appear less able to contribute towards understanding the wider and indirect costs associated with the disease. We explore several ways in which various sources of administrative data could enhance future research in this area.
\end{abstract}

\section{KEYWORDS}

administrative data, bowel cancer, colorectal cancer, costs, health economic research, systematic review

\section{1 | INTRODUCTION}

Colorectal cancer (CRC) is the third most common type of cancer globally, with over 1.8 million cases diagnosed in 2018. Incidence of CRC increases with age, and peak rates of new diagnoses occur in those aged 85-89. Furthermore, CRC accounts for the second highest number of deaths due to cancer. (CRUK, 2014).

Over the last forty years, technological advancements have enhanced detection and treatment for CRC, leading to improvements in survival and mortality rates (CRUK, 2014). In conjunction with the ageing population, the number of people living with and surviving cancer is expected to increase. In this way, CRC is increasingly considered a chronic condition, requiring care that stems beyond the initial phases of diagnosis and treatment.

The economic impact of better diagnosis, increasing lines of available treatment and improved survival is far reaching. Not only are there direct monetary implications for health and care service providers in terms of detection, treatment and follow-up care of 
CRC, but also there are indirect implications for patients, their families and wider society in terms of the impact of CRC on labour force participation and on both physical and mental well-being. It is crucial that we can measure these implications in order to assess the impact of CRC and to help inform policymakers decisions on how best to allocate a finite health budget.

The current availability of data to inform this understanding is somewhat limited and more often than not, data from clinical trials are used to make assumptions about the possible impact of an intervention on the entire population and ultimately inform decisions about resource allocation. Unfortunately, the generalisability of efficacy and cost-effectiveness measures from clinical trials to real-life populations can be limited by sample selection, size and attrition (He et al., 2020; Leon et al., 2006). Furthermore, clinical trials can be expensive to implement and run and often have short follow-up periods, meaning that longer term outcomes cannot be observed (Fitzpatrick et al., 2018).

One potential solution to the issue of the generalisability of trial data to whole populations lies in the use of administrative data. That is, data that are collected routinely 'by government departments and other organisations for the purposes of registration, transaction and record keeping, usually during the delivery of a service' (Woollard, 2014). Examples include hospital admissions data, education records and tax records. The routine collection of administrative data presents an exciting opportunity to conduct population level research that offers insights into healthcare resource use, costs and outcomes across a variety of domains such as education, income and retirement, through the linkage of these records to other data sets including clinical trials (Card et al., 2010; Einav \& Levin, 2014; Fitzpatrick et al., 2018). Moreover, administrative data can overcome the short follow-up period inherent in trials by tracking individuals over time, for example as they move in and out of hospital, into long-term care and even up to the end of their lives.

Despite these advantages, since administrative data are not generated for research purposes, they often lack the usual auxiliary measures that are used in social research to draw causal inference from a data set (Connelly et al., 2016). Thus, one of the central prospects for administrative data is for its use as a complementary source of information alongside clinical trials and survey data. The benefits of linking administrative records to observational data are documented elsewhere (Doiron et al., 2013).

Over the years, the potential of administrative data in research has been recognised worldwide and efforts have been made to harness that potential (Card et al., 2010; Einav \& Levin, 2014). In the Nordic countries in particular, robust data sharing infrastructures have been developed to facilitate researchers in making use of administrative data sets (Connelly et al., 2016). Moreover, the linkage aspect of administrative data has led to large data repositories emerging, where data sets are linked together and researchers can apply to access specific data sets and cohorts, to carry out their analysis (Doiron et al., 2013). Further, data repositories enhance research transparency because their indefinite storage allows for the replication of results. The success of such repositories has been made clear, for example the Western Australia Data Linkage System
(WADLS) repository includes over 30 population-based data sets and has produced over 250 journal publications (Doiron et al., 2013).

Of course, the creation of such repositories is not without its challenges. In particular, any research project that uses personal health data where informed consent is not obtained from patients may pose a risk to individual privacy. Therefore, central to the creation of a research repository is striking the appropriate balance between public benefit and patient privacy. That means being clear and transparent about the purposes of the research and its potential to generate patient or public benefit, at the same time taking measures to minimise the risk to patient privacy for example through the pseudonymisation or anonymisation of data.

We have identified that Scotland is in a unique position to demonstrate the potential contribution of administrative data, as well as an administrative data repository, within the health economic research of CRC. This is primarily due to the current data sharing and linkage infrastructure. Specifically, all Scottish residents have a unique Community Heath Index (CHI) number that permits the linkage of their administrative health records to one another and to other data sets.

The overarching aim of this paper is to investigate the potential contribution that administrative data could have on health economic research of CRC. To achieve this aim, the objectives were as follows:

1. To summarise the existing health economic research of CRC in the UK and Europe;

2. To identify whether and what types of administrative data were used within this research;

3. To explore the benefits and limitations of using administrative data in this research;

4. To discuss the ways in which administrative data, using Scotland as an exemplar, could contribute to this research in the future.

In what follows we outline the methods employed for the systematic review. Section 3 presents the results, and Section 4 discusses the findings and concludes.

\section{2 | METHODS}

\subsection{Search strategy}

We conducted a systematic literature search of Ovid MEDLINE R for English language articles published between 2009 and 2019. Specifically, our aim was to identify health economic research articles which focussed on CRC. To achieve this aim, we included those articles where the words 'colorectal cancer' or 'bowel cancer' appeared in the title and where both 'economic' and 'cost' appeared in the abstract. ${ }^{1}$

A Google Scholar search was also conducted to capture other relevant articles. We followed the PRISMA guidelines where applicable for conducting this review (PRISMA, 2020).

${ }^{1}$ The search strategy was as follows: 1 .(Colorectal cancer OR bowel cancer).title AND economic.abstract and cost.abstract. 2. Limit 1 to (English language AND year ="2009-2019"). 
TABLE 1 Definition of health economic studies included in final review

\begin{tabular}{|c|c|}
\hline Study & Description \\
\hline Budget Impact Analysis (BIA) & $\begin{array}{l}\text { Budget impact analyses assess the affordability of a novel healthcare intervention or policy change } \\
\text { applied to a specific healthcare budget, at an aggregate population level. }\end{array}$ \\
\hline $\begin{array}{l}\text { Cost Comparison (Cost } \\
\text { Minimisation) (CC) }\end{array}$ & $\begin{array}{l}\mathrm{CC} \text { is a method of comparing the costs of two or more interventions when the health outcomes of the } \\
\text { interventions are assumed to be the same. }\end{array}$ \\
\hline $\begin{array}{l}\text { Cost of Illness (Burden of Illness) } \\
\text { (COI) }\end{array}$ & $\begin{array}{l}\text { COI studies attempt to quantify the costs of a specific disease. This might be for the entire disease } \\
\text { pathway or for parts of it. Unlike EEs, they do not attempt to compare costs for competing } \\
\text { interventions rather, they provide an estimate of the cost given the existing provision of care. }\end{array}$ \\
\hline Economic Evaluation & $\begin{array}{l}\text { Economic evaluation aims to calculate the costs and benefits of an intervention or treatment, in order to } \\
\text { establish whether it is cost effective and thus inform investment in services. There are four main types } \\
\text { of economic evaluation, which differ in terms of how they measure outcomes: }\end{array}$ \\
\hline Cost Benefit Analysis (CBA) & In CBA, health outcomes are measured in monetary units. \\
\hline Cost-Effectiveness Analysis (CEA) & In CEA, outcomes are measured in natural or health units such as life years gained or cancers detected. \\
\hline Cost Utility Analysis (CUA) & $\begin{array}{l}\text { CUA is a special type of CEA in which outcomes are measured in preference based health outcomes such } \\
\text { as Quality Adjusted Life Years (QALY). }\end{array}$ \\
\hline
\end{tabular}

Source: Adapted from York Health Economics Consortium Glossary (York Health Economics Consortium, 2016)

FIGURE 1 Literature search results

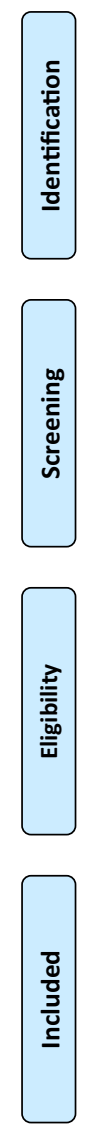

\subsection{Selection criteria}

Full-text publications of health economic studies were included when available in English language. The definitions of health economic studies are outlined in Table 1. Articles that were not carried out in Europe or the UK were excluded. Further, review articles were also excluded.
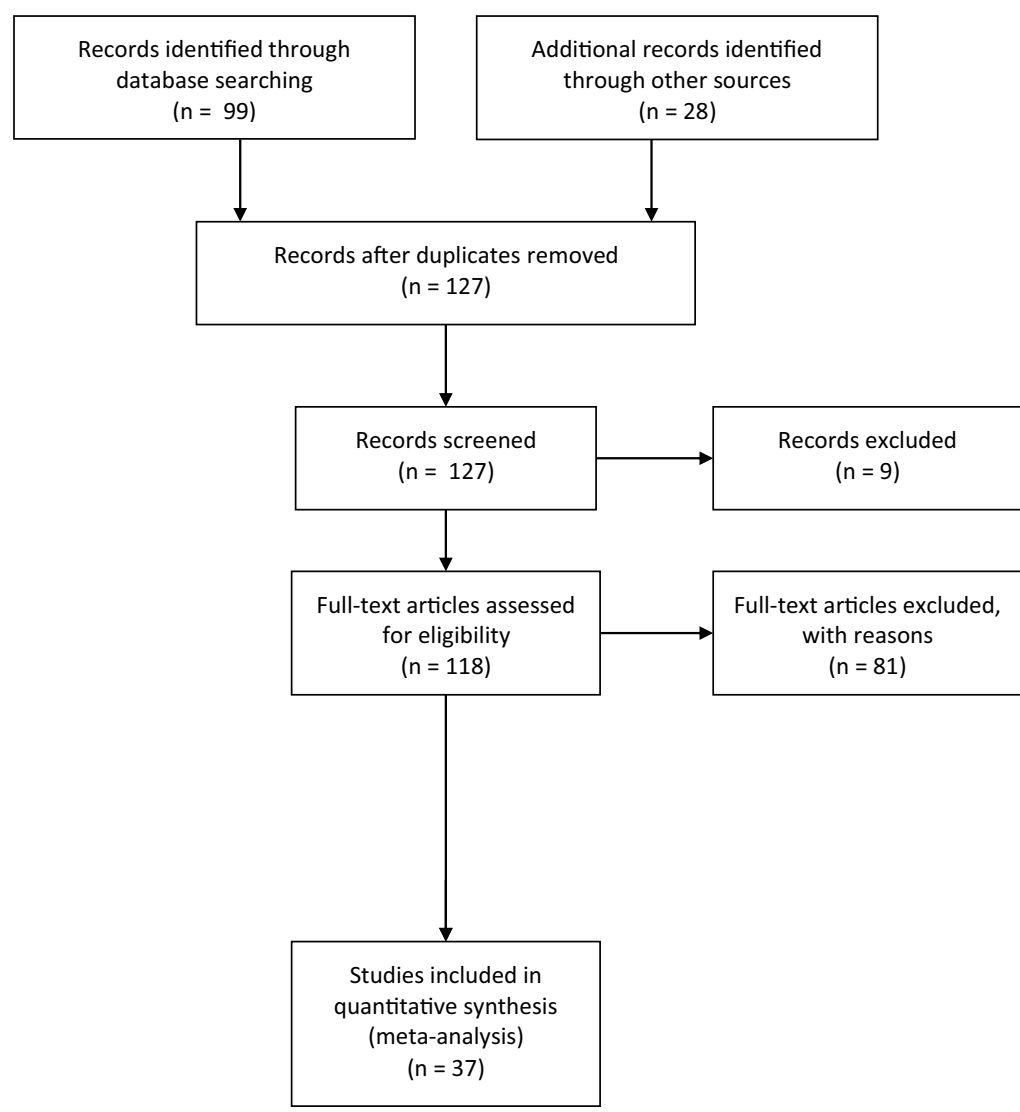

\section{3 | Data extraction}

The articles were grouped into the study groups as outlined in Table 1. A proforma was used to extract the relevant data from each article within these groups. For all types of studies, the country, perspective taken, method employed, data sources used (including administrative data), types of costs included, 
TABLE 2 UK/EU Economic Evaluations, $\mathrm{n}=19$

\begin{tabular}{|c|c|c|c|c|}
\hline Reference & Country & Perspective & Evaluation & Method \\
\hline Arrospide et al., (2018) & Basque & Healthcare system & CUA, BIA & Semi-Markov microsimulation \\
\hline Atkin et al., (2017) & UK & Health and social care system & CUA & Patient level simulation \\
\hline Asseburg et al., (2011) & Germany & Health insurance perspective & CEA & Patient level simulation \\
\hline Bullement et al., (2018) & England/Wales & Healthcare system & CEA, CUA & Partitioned survival model \\
\hline Halligan et al., (2015) & England & Healthcare system-secondary care & CEA & Descriptive \\
\hline Kearns et al., (2014) & England & Health and social care system & CEA, CUA & Markov model \\
\hline $\begin{array}{l}\text { Lansdorp-Vogelaar et al. } \\
\qquad(2018)\end{array}$ & Netherlands & Societal perspective & CEA & Semi-Markov microsimulation \\
\hline Matter-Walstra et al., (2016) & Switzerland & Healthcare system & CEA & Descriptive, regression \\
\hline Michalopoulos et al., (2013) & Greece & Not stated & CUA & Descriptive, regression \\
\hline Murphy et al., (2017) & England & Healthcare system & CUA, BIA & Markov model \\
\hline Pil et al., (2016) & Belgium & Societal perspective & CUA, BIA & Decision tree \& Markov model \\
\hline Pilgrim et al., (2009) & England & Not mentioned & CUA & Discrete event simulation \\
\hline Rao et al., (2018) & UK & Healthcare system & CUA & Decision tree $\&$ Markov model \\
\hline Rautenberg et al., (2014) & Germany & Health insurance perspective & CEA & Descriptive \\
\hline Robles-Zurita et al., (2018) & $\mathrm{UK}^{2}$ & Health and social care system & CUA & Partitioned survival model \\
\hline Sobhani et al., (2011) & France & Healthcare payer & CUA & Markov model \\
\hline Tilson et al., (2012) & Ireland & Healthcare payer & CEA & Decision tree model \\
\hline van der Meulen et al., (2018) & Netherlands & Third party payer & CUA & Semi-Markov microsimulation \\
\hline Whyte et al., (2012) & England & Health care system & CUA & State Transition Model \\
\hline
\end{tabular}

costs data sources used and the part of the CRC pathway under study were extracted. For the EEs, the type of evaluation was also noted.

Figure 1 below outlines the PRISMA (PRISMA, 2020) flow diagram of the search strategy results.

\section{3 | RESULTS}

\section{1 | Literature search results}

The articles were almost equally split between EEs $(n=19,51 \%)$ and costing studies $(n=18)$.

As per Table 1, the costing studies were categorised into Cost of Illness (COI) studies ( $\mathrm{n}=13,35 \%)$ and Cost Comparison (CC) studies $(n=5,14 \%)$.

\subsection{Summary of the existing health economic research of CRC in the UK and Europe}

Tables 2, 3 and 4 present the EEs, COI and CCs included in the final review.

Around $47 \%(n=9)$ of the EEs were conducted in the UK and the majority (68\%) of EEs conducted a CUA, whilst the remainder chose to implement a CEA. As is standard within an EE framework, the majority of studies accompany their EE with a decision tree, Markov model or simulation model, in order to extrapolate CRC costs and outcomes over time, for example up until the end of life.

In $\mathrm{EE}$, it is standard practice to explicitly state the perspective of the evaluation. Of the 19 EE's identified, 53\% $(n=10)$ take a healthcare system perspective. Other perspectives taken include a healthcare payer perspective, health insurance perspective or societal perspective. 


\begin{tabular}{|c|c|c|c|c|}
\hline Data Sources & Admin Data & Costs & Data Sources: Costs & Pathway \\
\hline $\begin{array}{l}\text { Screening data, cancer registry, } \\
\text { national statistics }\end{array}$ & Yes & Direct & Accounting system & Screening- end of life \\
\hline $\begin{array}{l}\text { Hospital records, previous studies, } \\
\text { life tables }\end{array}$ & Yes & Direct & National tariffs & Surveillance- end of life \\
\hline Previous studies, expert opinion & Yes (costs only) & Direct & National tariffs, market prices & Treatment - 10 years post \\
\hline Previous studies & Yes (costs only) & Direct & $\begin{array}{l}\text { Market prices, national tariffs, } \\
\text { previous study }\end{array}$ & Treatment- end of life \\
\hline Primary data collection & Yes & Direct & National tariffs, BNF & Diagnosis -5 years post \\
\hline Previous studies, screening data & Yes & Direct & Previous study & Screening- end of life \\
\hline Previous studies, life tables & No & Direct \& indirect & Previous studies & Screening- end of life \\
\hline Previous study & No & Direct & Primary data collection & $\begin{array}{l}\text { Treatment (and overall } \\
\text { survival) }\end{array}$ \\
\hline Primary data collection & No & Direct & Primary data collection & Surgery \\
\hline Previous studies, life tables & Yes (costs only) & Direct & $\begin{array}{l}\text { National tariffs, screening data, } \\
\text { previous study }\end{array}$ & Screening-end of life \\
\hline $\begin{array}{l}\text { Previous studies, cancer registry, } \\
\text { screening data, national } \\
\text { statistics }\end{array}$ & Yes & Direct \& indirect & $\begin{array}{l}\text { Official Belgian costs of medical } \\
\text { procedures, previous studies }\end{array}$ & Screening - 50 years post \\
\hline $\begin{array}{l}\text { Previous studies, hospital records, } \\
\text { expert opinion }\end{array}$ & Yes & Direct & National tariffs, previous studies & Screening- end of life \\
\hline $\begin{array}{l}\text { Previous studies, hospital records, } \\
\text { life tables }\end{array}$ & Yes & Direct & National tariffs & Treatment- end of life \\
\hline Previous studies & No & Direct & Market prices & Treatment \\
\hline Previous study & Yes (costs only) & Direct & $\begin{array}{l}\text { National tariffs, primary } \\
\text { collection }\end{array}$ & Treatment- 8 years post \\
\hline Previous studies, national statistics & No & Direct & Previous studies & Screening - end of life \\
\hline $\begin{array}{l}\text { Previous studies, cancer registry, } \\
\text { hospital records, expert opinion }\end{array}$ & Yes & Direct & $\begin{array}{c}\text { National tariffs, previous studies, } \\
\text { hospital finance department }\end{array}$ & Diagnosis -5 years post \\
\hline Previous studies, life tables & No & Direct & Previous study & Screening- end of life \\
\hline $\begin{array}{l}\text { Previous studies, screening data, } \\
\text { expert opinion }\end{array}$ & Yes & Direct & National tariffs, previous study & Screening- end of life \\
\hline
\end{tabular}

The perspective taken influences the types of costs that are included. As a result, the vast majority (89\%) of studies only include direct costs associated with the delivery of care. The two studies which take a societal perspective, Lansdorp-Vogelaar et al., (2018) and Pil et al., (2016), also incorporate indirect costs, that is additional costs encountered by the patients such as loss of earnings. In terms of which part of the CRC pathway is investigated, the most common evaluations are conducted on screening programmes. In particular, $45 \%(n=9)$ of the included studies evaluate the cost-effectiveness of different CRC screening programmes. A further $40 \%(n=7)$ look at the cost-effectiveness of treatment for CRC, including curative treatment and treatment for metastatic disease. A smaller proportion of the EE's, $10 \%(n=2)$, look at diagnosis and $5 \%(n=1)$ at surveillance of adenomas. As most studies use Markov and microsimulation models, they tend to model outcomes and costs beyond the initial pathway starting point, either until the end of life or an alternative long-term end point, for example 50 year follow-up. In addition to conducting a CUA to assess the value of a healthcare intervention, three of the EEs conducted a budget impact analysis (BIA) to assess the affordability of the intervention for a specific healthcare budget (Arrospide et al., 2018; Murphy et al., 2017; Pil et al., 2016).

In contrast to the evaluations, only $31 \%(n=4)$ of the COI articles identified were UK based (England only). Ireland accounted for almost a quarter of the studies $(n=3)$, followed by Italy $(n=2)$, France $(n=2)$ and Spain $(n=2)$. The majority of COI articles conducted retrospective cohort analyses (77\%). This involves looking at historical data to identify a cohort of patients, for example those with metastatic CRC, and costing their use of healthcare resources.

In addition, COI studies were less likely to mention which perspective the analysis is conducted from. However, like EEs, the COI studies tended to focus on direct costs. Only two COI papers looked solely at indirect costs (Hanly et al., 2013; ÓCéilleachair et al., 2017) and one included both direct and indirect costs (Lejeune et al., 2009). With respect to the particular part of the CRC care pathway costed, 


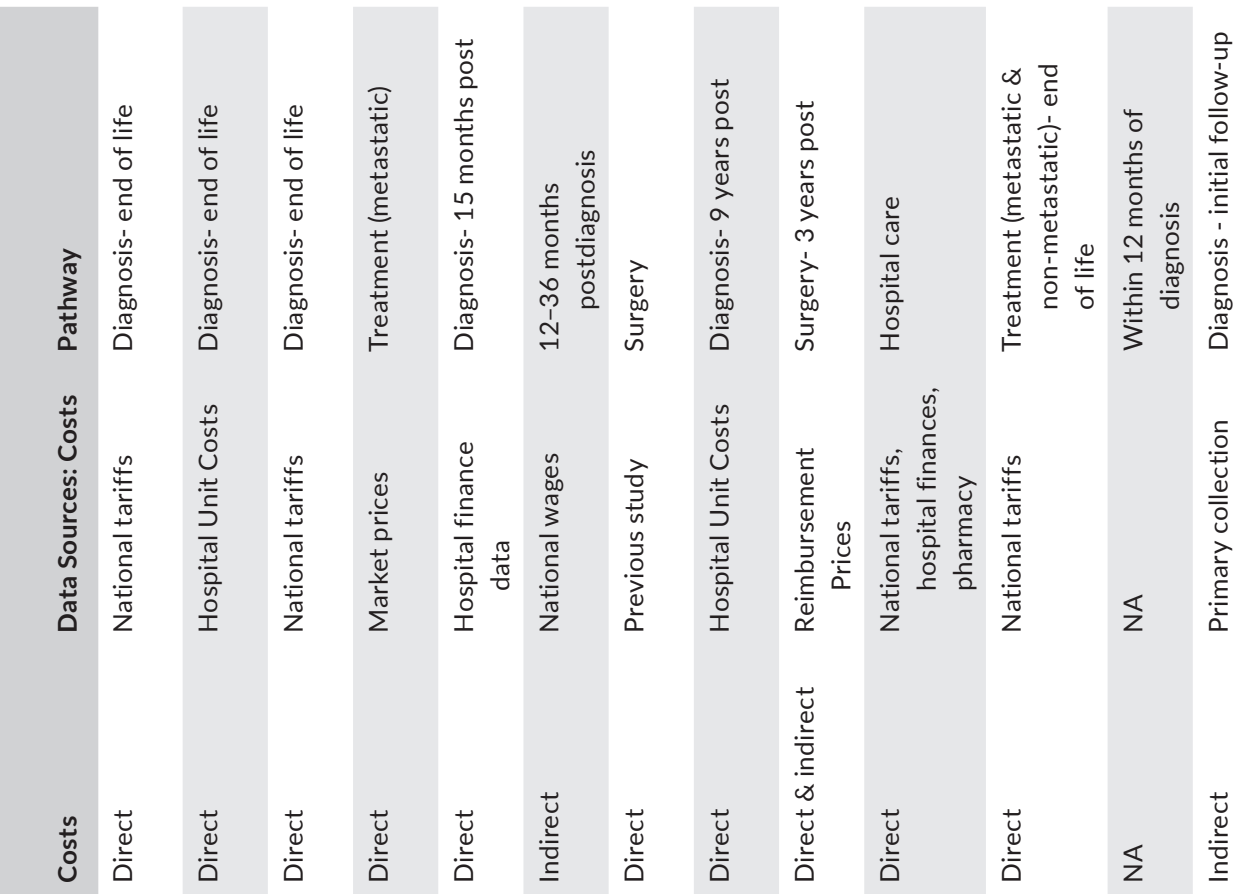

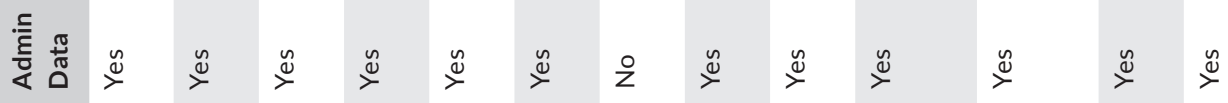
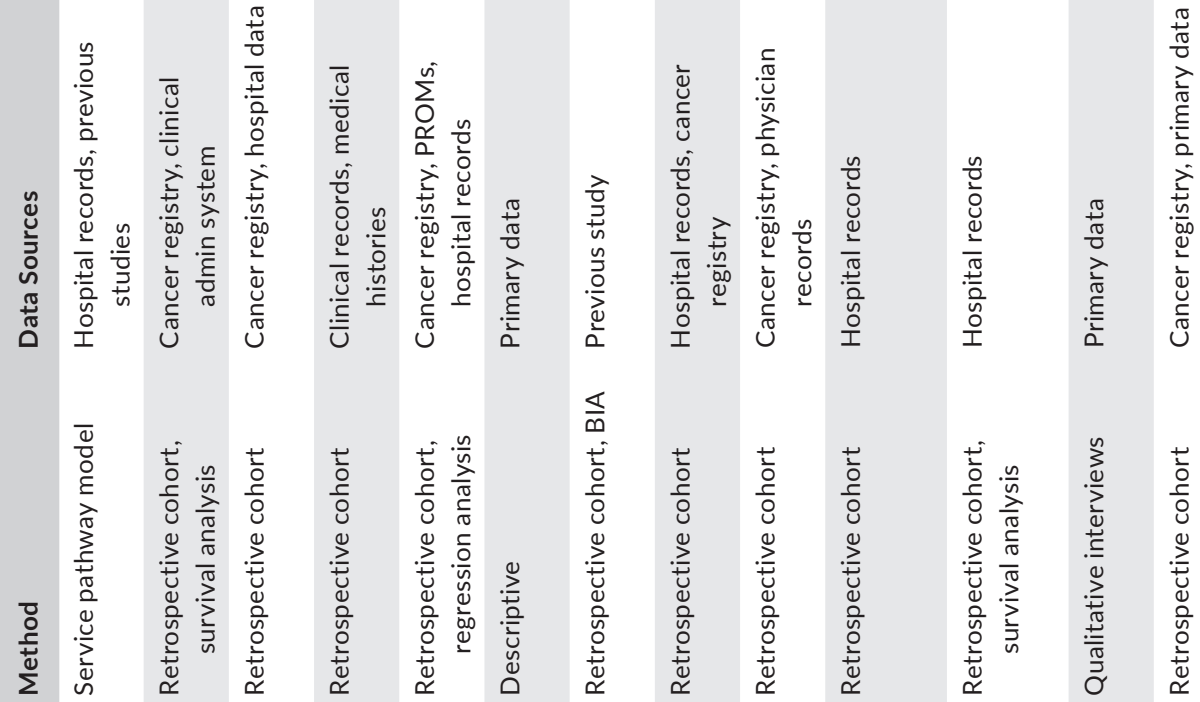

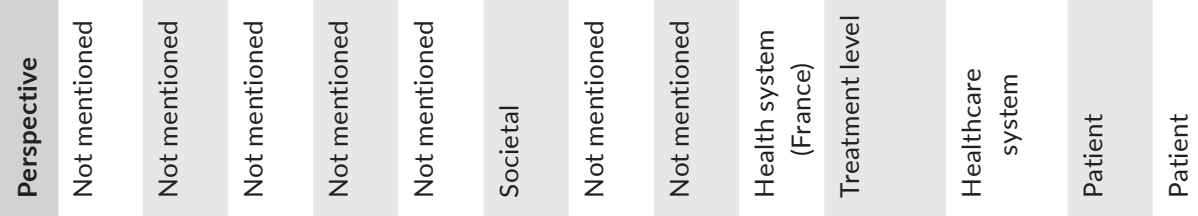

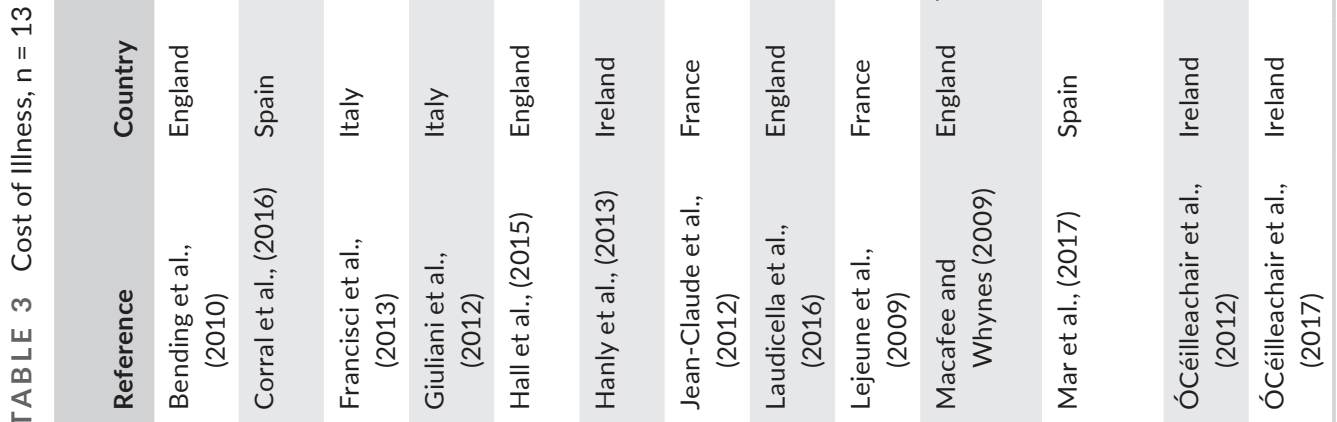




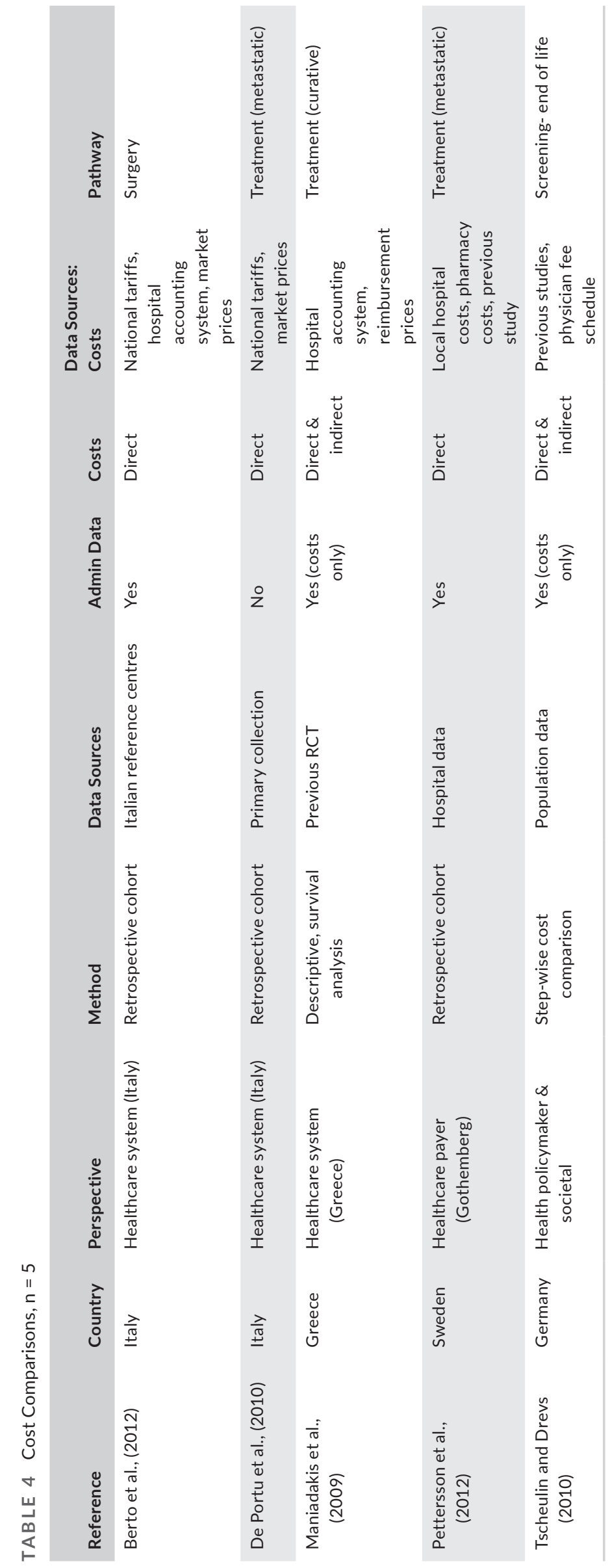


TABLE 5 Administrative data sources

\begin{tabular}{|c|c|}
\hline Data source & Description \\
\hline Cancer registry data & $\begin{array}{l}\text { Cancer registries contain a record of all cases of new cancer diagnoses in one centralised system. They tend } \\
\text { to include information on cancer diagnoses and treatment, allowing a country to monitor cancer incidence } \\
\text { and survival, and any emerging trends, over a long period of time. Registries also include patient level } \\
\text { demographics, permitting analyses of diagnoses by age, gender and stage distribution. They can also include } \\
\text { information on cancer related mortality. }\end{array}$ \\
\hline Screening programme data & $\begin{array}{l}\text { Screening programme data sets provide a wealth of information including participation and compliance rates, } \\
\text { adenoma and } \mathrm{CRC} \text { detection rates, specificity and sensitivity, as well as information on surveillance. In some } \\
\text { cases, follow-up data are also available, for example on colonoscopies and flexible sigmoidoscopy. Follow-up } \\
\text { data provide information on participation, detection and complications. }\end{array}$ \\
\hline Routine hospital records & $\begin{array}{l}\text { Routine hospital records provide information on any acute hospital admission experienced by a patient, including } \\
\text { length of stay and procedure codes. Moreover, hospital records often include additional information about an } \\
\text { individuals primary and secondary diagnoses, allowing the researcher to gather more information about patient } \\
\text { co-morbidity and other procedures and medications related to or unrelated to their cancer diagnosis. }\end{array}$ \\
\hline Costs databases & $\begin{array}{l}\text { Administrative costs data are collected in the form of national tariffs for the reimbursement of the provision of } \\
\text { hospital services and in hospital accounting systems. These systems are usually updated annually and therefore } \\
\text { provide robust and up to date estimates of unit costs for economic analyses. }\end{array}$ \\
\hline
\end{tabular}

there was considerable variation within the COI studies. Three looked at the pathway from diagnosis to end of life (Bending et al., 2010; Corral et al., 2016; Francisci et al., 2013). Others focussed on diagnosis, but only looked at costs from diagnosis up to a pre-specified time point, for example 12 months postdiagnosis or within 12 months of initial diagnosis. Two studies looked at all hospital care throughout the care pathway (Laudicella et al., 2016; Macafee \& Whynes, 2009). One study focussed on treatment of metastatic and non-metastatic disease up until the end of life (Mar et al., 2017), whilst another focussed on costs of treating metastatic disease alone (Giuliani et al., 2012). Similarly, one study looked at the cost of surgery alone (JeanClaude et al., 2012)and another at the costs from surgery up to three years post-surgery (Lejeune et al., 2009). One COI study also conducted a BIA for patients who underwent CRC surgery in French nonprofit hospitals (Jean-Claude et al., 2012).

Of the CC studies identified in Table 4, two of the CC studies are from Italy and the remaining three are from Greece, Sweden and Germany. As with the COI studies, the predominant methodology applied in the CCs is a retrospective cohort approach. In terms of the perspective, the majority of the $\mathrm{CC}$ studies conduct their analyses from the perspective of the health system in which they are based. One of the studies takes the perspective of the healthcare payer, and another takes both a health policymaker and a societal perspective. Once again, the focus on costs is mainly on direct costs; however, two CC papers also incorporate indirect costs. The majority of CC studies focus on the treatment part of the CRC pathway. One paper focusses on surgery and another on screening up until the end of life.

\section{3 | Types of administrative data used within the health economic research of CRC in the UK and Europe}

The papers identified use a mixture of data sources including administrative data, national statistics, previous studies including randomised control trials (RCTs), expert opinion and in some cases primary data collection. Table 5 below outlines the administrative data sources that appeared most frequently in the studies. In addition, Tables 6, 7 and 8 in the appendix provide a broader overview of the various data sources used to inform the main groups of parameters included in the literature.

Within the EEs, $68 \%(n=13)$ utilise administrative data. The most common use of administrative data within these studies is via the use of administrative costs data bases to calculate direct costs (those which relate directly to patient care such as a hospital stay). Further, some studies use administrative data, for example cancer registry, to inform particular patient and clinical parameters in the decision trees, Markov and simulation models. Of the EEs which use administrative data, five evaluate screening programmes and use administrative screening programme data in their analysis. This clearly reflects the effort in many European countries in recent years to detect cancer as early as possible for those at the highest risk by rolling out national screening programmes for CRC. As a result, a multitude of administrative screening data sets have been created and researchers have capitalised on this opportunity.

It is also common for those studies to combine the screening data with other administrative data sets. In particular, Arrospide et al., (2018) combine data from the Basque screening programme with cancer registry data to evaluate the Basque CRC screening programme. Pil et al., (2016) use data from the Belgian government screening programme alongside the Belgian cancer registry in their analysis of a population-based CRC screening programme. Finally, in their evaluation of different surveillance strategies for patients with intermediate-grade adenomas, Atkin et al., (2017) use routine hospital records together with data from the English bowel cancer screening pilot. In the three EEs (Arrospide et al., 2018; Murphy et al., 2017; Pil et al., 2016) who undertook BIAs, location-specific estimates of population size, age-specific disease incidence, resource use and location-specific costs were acquired from various administrative 
data sources to permit analyses that were relevant and useful to the budget holder in question.

At the same time, the extent to which administrative data are used within the EEs varies considerably and no one study relies exclusively on routine data. For example, Atkin et al., (2017) use routine hospital records linked to cancer registry data to inform many of the parameters in their patient level simulation model, whilst Rao et al., (2018) use routine hospital records solely for the purposes of informing their parameter on postoperative mortality. Furthermore, several EEs use administrative costs data only (Asseburg et al., 2011; Bullement et al., 2018; Murphy et al., 2017; Robles-Zurita et al., 2018). In every EE, the existing literature or previous RCTs are also used to inform specific model parameters. Where this is the case, it is possible that the prior research also used administrative data.

The COI studies utilise administrative data more often compared to the EEs. In particular, 11 of the 12 COI papers identified in Table 3 use administrative data. In comparison with the EE's, where much of the administrative data comes from screening programmes, the main sources of administrative data in the $\mathrm{COI}$ studies come from cancer registries and routine hospital records. In the majority of cases, the $\mathrm{COI}$ studies use cancer registry data to identify a cohort of patients to be analysed. The registry data provide clinical information on CRC diagnosis, staging, location of tumour, date of diagnosis etc. This information is then linked to routine hospital records that contain information on treatment, co morbidities, complications and recurrence etc.

In addition to using cancer registries and clinical information systems to analyse CRC cohorts, several $\mathrm{CO}$ studies use registry data as a means of identifying patients to invite them to participate in a survey or interview. Specifically, Hanly et al., (2013) and ÓCéilleachair et al., (2017) use Irish cancer registry data to identify individuals with primary, invasive CRC in order to invite them to complete postal questionnaires. Survey responses are then combined with clinical information from the cancer registry to conduct statistical analysis. Similarly, ÓCéilleachair et al., (2012) used hospital records from six participating sites to identify patients who would be eligible to take part in interviews for their qualitative analysis of the inter-relationships between the economic and emotional consequences of CRC.

Like EE's, COI studies often utilise administrative costs data bases to calculate direct costs. Only one study used administrative data to inform the calculation of indirect costs (those costs that occur outside the delivery of patient care, such as lost productivity or foregone wages). Specifically, Lejeune et al., (2009) use hospital records data to measure the distance travelled to and from the patients home to consult with their GP or gastroenterologist, which was then used to calculate indirect costs to the patient.

Overall, compared to EEs, COI studies are more likely to rely exclusively on administrative data (Corral et al., 2016; Francisci et al., 2013; Giuliani et al., 2012; Laudicella et al., 2016; Lejeune et al., 2009; Macafee \& Whynes, 2009; Mar et al., 2017) and are far less likely to use previous studies to inform parameters.
Finally, almost all of the CC studies use administrative data of some sort. As with the EEs, some use administrative data in the form of costs databases only and like the COI studies some use administrative hospital records.

\section{DISCUSSION AND CONCLUSION}

Clearly, one area in which administrative data have been particularly powerful is in evidence on the cost-effectiveness of various screening strategies for CRC, which has resulted from the evolution of national screening programmes throughout Europe. Data from these programmes have been used to inform and update many of the crucial parameters used in the models that accompany EEs of screening programmes. This evidence base invariably demonstrates the feasibility and potential of collecting administrative data on this scale to inform other parts of the treatment pathway for CRC.

At the same time, administrative cancer registry data have proved to be useful in terms of defining and identifying cohorts for costing studies and again for informing vital parameters such as disease prevalence, treatment and outcomes. Many EE's have also taken advantage of the power of data linkage by linking administrative records to data form participants in RCTs.

Furthermore, since providing estimates of costs is central to conducting both EE's and costing analyses, the emergence of costs databases have proved to be a valuable source of information on costs for all areas of economic research into CRC. Specifically, 43\% $(n=16)$ of the studies identified used administrative costs databases.

The administrative costs databases have proved particularly powerful in the studies that include direct costs. In particular, the costing approaches implemented in those papers are consistent with the existence of European Disease Related Group (DRG) type systems for reimbursing hospitals for their services. Therefore, unsurprisingly, many of them implement a 'top-down' costing approach by using national tariffs based on DRGs to attach monetary values to patients resource utilisation (Špacírová et al., 2020). This highlights the potential for administrative data to contribute to understanding the costs of delivering CRC care.

Finally, the merit of using administrative data for the purposes of $\mathrm{BIA}$ is clear. In an era of increasing austerity and budget cuts, using administrative data within BIAs to more accurately predict the affordability of introducing novel interventions into a fixed budget healthcare system will ensure more efficient allocation of resources. Using locally or nationally collected administrative data for the purposes of BIA is particularly useful because this will make any analysis more relevant and useful to the budget holder in question.

Having said that, we have identified some areas where the use of administrative data has been limited. For example, although one of the main advantages of using routine records in research is their ability to capture large populations over long periods of time, we find little evidence that this is the case for the health economics literature on CRC. Specifically, only one costing study used routine records to capture an entire population over a long period of time 
(Laudicella et al., 2016). Excluding this example, the maximum sample size identified is less than a few thousand and in most cases, the populations under study come from a single hospital or administrative area. At the same time, many of the costing studies identified look at one specific part of the disease pathway with a limited follow-up period. Overall, it appears that the power of administrative data to provide evidence for whole populations, spanning the entire disease pathway and follow-up for survivors, is yet to be harnessed.

Related to this, we found a lack of evidence on the wider costs associated with CRC, particularly with respect to social care and indirect costs such as unpaid care. For example, although evidence shows that many cancer patients need social care as a direct consequence of their condition and the consequences of its treatment, none of the papers identified look at the use of social care services by CRC patients (MacMillan Cancer Support, 2015).

Furthermore, few papers explored indirect costs. In particular, only two EEs explicitly take a societal perspective and therefore include both direct and indirect costs of care (LansdorpVogelaar et al., 2018; Pil et al., 2016). Within the COI studies, Hanly et al., (2013) and ÓCéilleachair et al., (2017) focus exclusively on indirect costs, whilst Lejeune et al., (2009) include direct and indirect costs. Further, the CC's carried out by Maniadakis et al., (2009) and Tscheulin and Drevs (2010) also include both direct and indirect costs. The lack of inclusion of indirect costs overall is not surprising given that they are notoriously difficult to measure. However, of those who did, the use of administrative data was even less likely. Clearly, measuring indirect costs is challenging in itself, but in addition to this, the administrative data appear less able to contribute to studies which include the indirect costs of CRC. This highlights a key limitation of administrative records in their ability to capture indirect costs.

Finally, it appears that administrative data are less able to contribute when it comes to measuring patient health related quality of life (HRQoL) and preferences for those health states, which is vital particularly in EEs. None of the EEs used administrative data to inform utility parameters. In most cases, studies look to previous literature, often going back several years, for this information. As administrative data are not collected for the purposes of research, it is not surprising that they lack the types of measures needed to capture patient outcomes in the way that is needed for EEs. Nonetheless, recent developments in tools to capture Patient Reported Outcome Measures (PROMS) on symptoms, condition and quality of life might be used to measure outcomes in CRC patients. PROMS will add a valuable patient-centred dimension to routine data sets, and plans are underway to collect this data in the future.

In summary, it is not uncommon for the health economic research of CRC to utilise administrative records to aid EEs and costing analyses, and undoubtedly, they can offer a wealth of information about an individuals CRC diagnoses, subsequent treatment and follow-up over time. However, there appear to be several limitations to their use and gaps in the existing evidence. In what follows, we consider how Scottish administrative data might mitigate those limitations and fill in some of the gaps in the evidence.
As discussed in Section 1, Scotland is in a prime position to demonstrate the contribution of administrative data, in particular due to its data sharing and data linkage infrastructure. In theory, this infrastructure means that all health data sets can easily be linked to one another and to administrative data sets in other domains, for example social care.

In October 2018, the Public Benefit Privacy Panel for health and social care approved a project to link several administrative data sets for CRC patients in Scotland (Study numer: 1718-0026), in order to conduct research into the economics of CRC. This project is part of a wider Cancer Research UK funded project, Bowel Cancer Intelligence UK, which has been granted permission from the Research Ethics Service of the Health Research Authority for a CRC research data repository called COloRECTal Repository (CORECT-R) (BCIUK, 2021). The Scottish data provide a useful platform on which to demonstrate the possible contribution of administrative data and a CRC repository to the health economic research of CRC within the UK and Europe. A contribution that is enhanced by the similarities between data collection and data coding systems within the constituent UK nations and further afield, for example, the International Classification of Disease (ICD) and OPCS Classification of Interventions and Procedures (OPCS-4). This provides the opportunity for research conducted in a Scottish CRC context to be replicated with similar linkages outside of Scotland. However, further work will be required to harmonise data sets to allow for cross country comparisons. This may be done, for example, in line with the Observational Medical Outcomes Partnership (OMOP) Common Data Model (Observational Health Data Sciences and Informatics, 2021).

Furthermore, Scottish administrative data could contribute to the evidence by simply providing evidence for Scotland. Despite the wealth of administrative data sets and data infrastructure in existence in Scotland, there appears to be limited health economics research of CRC within the Scottish context. In particular, only two studies identified in this review used Scottish administrative records (Atkin et al., 2017; Robles-Zurita et al., 2018). In both studies, Scotland was represented alongside data from other countries. Thus, a Scottish CRC data repository would afford the opportunity for health economic research into CRC in Scotland to be realised.

In addition, the use of Scottish administrative data could be used to inform and update common model parameters used in both EEs and costing studies, using data that reflects current practice for an entire population, for example, prevalence and incidence rates, durations of treatments, survival outcomes etc. This information could not only be useful for other health economic studies of CRC in Scotland, but also for other nations in the UK and potentially other European countries who have similar demographics and health systems.

The data linkage infrastructure in Scotland would mean that all relevant health data sets can be linked to one another, in particular, cancer registry, cancer treatment, screening, outpatient and inpatient, prescriptions, accident and emergency, GP data and more. This level of information would potentially capture the patients entire 
CRC journey through the healthcare system, pre- and postdiagnosis, allowing for the more precise measurement of the key inputs into health economic studies of CRC. Furthermore, health data sets can also be linked to other administrative databases like social care and Department for Work and Pensions (DWP) data. These linkages to other administrative data sets outwith the healthcare system could provide additional information about a patients experience which could again be used to inform health economic research in this area, both within and outwith Scotland.

Finally, and related to the linkage opportunities, the use of Scottish administrative data could enhance the evidence base on other direct and indirect costs related to CRC. Specifically, the linkage to social care records could be particularly powerful in this respect. As highlighted earlier, none of the studies we identified looked at the use of longer term social care services by CRC patients, despite existing evidence showing that many cancer patients need social care as a direct consequence of their condition and the consequences of its treatment (MacMillan Cancer Support, 2015). In Scotland, local authorities are required to routinely collect information on all social care services delivered to people within their area. This data could be used to provide evidence on other nonhealth related direct costs associated with CRC, again both during treatment and beyond. In addition, as part of the social care data collection, an indicator of the presence of an unpaid carer is collected for social care clients. This information could be useful for understanding the indirect costs associated with a CRC diagnosis, in terms of the reliance on unpaid carers to provide additional care and support.

Going forward, it is important to recognise that there are questions administrative data cannot answer alone. In such cases, trial and survey data may fill the gaps, and vice versa. Specifically, clinical trial data offer the opportunity for randomisation, blinding and/or stratification, which allow assessment of the efficiency of new or existing treatments in a highly selected group of patients. In contrast, administrative data can play a unique role in testing the effectiveness, in a real world setting, of treatments that have already been tested within trials. This sequence of events means that the types of questions that trial data set out to answer are likely to be different to those using administrative data. Overall, administrative data do not remove the need for trial or other data sources, instead these sources of data are complimentary.

That being said, we have found that the use of administrative data is common within the UK and EU health economic research on CRC. In particular, cancer registry, screening and routine hospital records were commonly used. In the EE's, administrative data tended to be supplemented with data from the clinical trial under study and/ or from the existing literature. Costing studies were more likely to rely heavily on administrative records. Overall, we find that although administrative data are present, they do not appear to being used to their full potential and administrative data, including data repositories, within the UK and Europe could have a significant impact on research in this area. Scotland, in particular, may provide a valuable exemplar to unlock this potential.

\section{CONFLICT OF INTEREST}

The authors have no conflict of interest to report.

\section{DATA AVAILABILITY STATEMENT}

Data sharing not applicable to this article as no data sets were generated or analysed during the current study.

\section{ORCID}

Elizabeth Lemmon (D) https://orcid.org/0000-0002-3564-6106

Catherine R. Hanna (D) https://orcid.org/0000-0002-0907-7747

Peter Hall D https://orcid.org/0000-0001-6015-7841

Eva J. A. Morris (D) https://orcid.org/0000-0002-1075-6544

\section{REFERENCES}

Arrospide, A., Idigoras, I., Mar, J., de Koning, H., van der Meulen, M., Soto-Gordoa, M., Martinez-Llorente, J. M., Portillo, I., Arana-Arri, E., Ibarrondo, O., \& Lansdorp-Vogelaar, I. (2018). Costeffectiveness and budget impact analyses of a colorectal cancer screening programme in a high adenoma prevalence scenario using miscancolon microsimulation model. BMC Cancer, 18, 464. https://doi. org/10.1186/s12885-018-4362-1

Asseburg, C., Frank, M., Köhne, C. H., Hartmann, J. T., Griebsch, I., Mohr, A., Osowski, U., Schulten, J., \& Mittendorf, T. (2011). Costeffectiveness of targeted therapy with cetuximab in patients with k-ras wild-type colorectal cancer presenting with initially unresectable metastases limited to the liver in a german setting. Clinical Therapeutics, 33, 482-497. https://doi.org/10.1016/j.clint hera.2011.04.010

Atkin, W., Brenner, A., Martin, J., Wooldrage, K., Shah, U., Lucas, F., Greliak, P., Pack, K., Kralj-Hans, I., Thomson, A., Perera, S., Wood, J., Miles, A., Wardle, J., Kearns, B., Tappenden, P., Myles, J., Veitch, A., \& Duffy, S. W. (2017). The clinical effectiveness of different surveillance strategies to prevent colorectal cancer in people with intermediate-grade colorectal adenomas: a retrospective cohort analysis, and psychological and economic evaluations. Health Technology Assessment, 21(25), 1-536. https://doi.org/10.3310/ hta21250

BCIUK(2021). Bowel Cancer Intelligence UK website, https://bci.leeds. ac.uk/. Accessed on 02 June, 2021

Bending, M. W., Trueman, P., Lowson, K. V., Pilgrim, H., Tappenden, P., Chilcott, J., \& Tappenden, J. (2010). Estimating the direct costs of bowel cancer services provided by the national health service in england. International Journal of Technology Assessment in Health Care, 26, 362-369. https://doi.org/10.1017/S0266462310001078

Berto, P., Lopatriello, S., Aiello, A., Corcione, F., Spinoglio, G., Trapani, V., $\&$ Melotti, G. (2012). Cost of laparoscopy and laparotomy in the surgical treatment of colorectal cancer. Surgical Endoscopy, 26, 14441453. https://doi.org/10.1007/s00464-011-2053-y

Bullement, A., Underhill, S., Fougeray, R., \& Hatswell, A. J. (2018). Costeffectiveness of trifluridine/tipiracil for previously treated metastatic colorectal cancer in england and wales. Clinical Colorectal Cancer, 17, e143-e151. https://doi.org/10.1016/j.clcc.2017.09.001

Card, D., Chetty, R., Feldstein, M. S., \& Saez, E. (2010). Expanding access to administrative data for research in the united states. American Economic Association, Ten Years and Beyond: Economists Answer NSF's Call for Long-Term Research Agendas.

Connelly, R., Playford, C. J., Gayle, V., \& Dibben, C. (2016). The role of administrative data in the big data revolution in social science research. Social Science Research, 59, 1-12. https://doi.org/10.1016/j. ssresearch.2016.04.015

Corral, J., Castells, X., Molins, E., Chiarello, P., Borras, J. M., \& Cots, F. (2016). Long-term costs of colorectal cancer treatment in spain. 
BMC Health Services Research, 16, 56. https://doi.org/10.1186/ s12913-016-1297-6

CRUK. (2014). Cancer research uk, bowel cancer incidence statistics (2014-2016). https://edin.ac/32Abfal, Accessed July 20, 2020.

De Portu, S., Mantovani, L., Ravaioli, A., Tamburini, E., Bollina, R., Cozzi, C., Grimaldi, A., Testa, T., Bianchessi, C., \& Cartenì, G. (2010). Cost analysis of capecitabine vs 5 -fluorouracilbased treatment for metastatic colorectal cancer patients. Journal of Chemotherapy, 22, 125128. https://doi.org/10.1179/joc.2010.22.2.125

Doiron, D., Raina, P., \& Fortier, I. (2013). Linking canadian population health data: maximizing the potential of cohort and administrative data. Canadian Journal of Public Health, 104, e258-e261. https:// doi.org/10.17269/cjph.104.3775

Einav, L., \& Levin, J. (2014). The data revolution and economic analysis. Innovation Policy and the Economy, 14, 1-24. https://doi. org/10.1086/674019

Fitzpatrick, T., Perrier, L., Shakik, S., Cairncross, Z., Tricco, A. C., Lix, L., Zwarenstein, M., Rosella, L., \& Henry, D. (2018). Assessment of long-term follow-up of randomized trial participants by linkage to routinely collected data: a scoping review and analysis. JAMA Network Open, 1, e186019. https://doi.org/10.1001/jamanetwor kopen.2018.6019

Francisci, S., Guzzinati, S., Mezzetti, M., Crocetti, E., Giusti, F., Miccinesi, G., Paci, E., Angiolini, C., \& Gigli, A. (2013). Cost profiles of colorectal cancer patients in italy based on individual patterns of care. BMC Cancer, 13, 329. https://doi.org/10.1186/1471-2407-13-329

Giuliani, J., Indelli, M., Marzola, M., Raisi, E., \& Frassoldati, A. (2012). The management of skin toxicity during cetuximab treatment in advanced colorectal cancer: How much does it cost? a retrospecive economic assessment from a single-center experience. Tumori Journal, 98, 408-412. https://doi.org/10.1177/030089161209800402

Hall, P. S., Hamilton, P., Hulme, C. T., Meads, D. M., Jones, H., Newsham, A., Marti, J., Smith, A. F., Mason, H., Velikova, G., Ashley, L., \& Wright, P. (2015). Costs of cancer care for use in economic evaluation: A UK analysis of patient-level routine health system data. British Journal of Cancer, 112, 948. https://doi.org/10.1038/bjc.2014.644

Halligan, S., Dadswell, E., Wooldrage, K., Wardle, J., von Wagner, C., Lilford, R., Yao, G. L., Zhu, S., \& Atkin, W. (2015). Computed tomographic colonography compared with colonoscopy or barium enema for diagnosis of colorectal cancer in older symptomatic patients: two multicentre randomised trials with economic evaluation (the siggar trials). Health Technology Assessment, 19(54), 1-134. https://doi.org/10.3310/hta19540

Hanly, P., Céilleachair, A. Ó., Skally, M., O'Leary, E., Staines, A., Kapur, K., Fitzpatrick, P., \& Sharp, L. (2013). Time costs associated with informal care for colorectal cancer: an investigation of the impact of alternative valuation methods. Applied Health Economics and Health Policy, 11, 193-203. https://doi.org/10.1007/s40258-013-0013-5

He, Z., Tang, X., Yang, X., Guo, Y., George, T. J., Charness, N., Quan Hem, K. B., Hogan, W., \& Bian, J. (2020). Clinical trial generalizability assessment in the big data era: A review. Clinical and Translational Science, 13, 675-684. https://doi.org/10.1111/cts.12764

Jean-Claude, M., Emmanuelle, P., Juliette, H., Michèle, B., Gérard, D., Eric, F., Xavier, H., Bertrand, L., Jean-Fabien, Z., Yves, P., \& Gérard, N. (2012). Clinical and economic impact of malnutrition per se on the postoperative course of colorectal cancer patients. Clinical Nutrition, 31, 896-902. https://doi.org/10.1016/j.clnu.2012.03.011

Kearns, B., Whyte, S., Chilcott, J., \& Patnick, J. (2014). Guaiac faecal occult blood test performance at initial and repeat screens in the english bowel cancer screening programme. British Journal of Cancer, 111, 1734. https://doi.org/10.1038/bjc.2014.469

Lansdorp-Vogelaar, I., Goede, S. L., Bosch, L. J., Melotte, V., Carvalho, B., van Engeland, M., Meijer, G. A., de Koning, H. J., \& van Ballegooijen, M. (2018). Cost-effectiveness of highperformance biomarker tests vs fecal immunochemical test for noninvasive colorectal cancer screening. Clinical Gastroenterology and Hepatology, 16, 504-512.
Laudicella, M., Walsh, B., Burns, E., \& Smith, P. C. (2016). Cost of care for cancer patients in england: evidence from population-based patient-level data. British Journal of Cancer, 114, 1286-1292. https://doi.org/10.1038/bjc.2016.77

Lejeune, C., Binquet, C., Bonnetain, F., Mahboubi, A., Abrahamowicz, M., Moreau, T., Raikou, M., Bedenne, L., Quantin, C., \& BonithonKopp, C. (2009). Estimating the cost related to surveillance of colorectal cancer in a french population. The European Journal of Health Economics, 10, 409-419. https://doi.org/10.1007/s1019 8-009-0144-7

Leon, A. C., Mallinckrodt, C. H., Chuang-Stein, C., Archibald, D. G., Archer, G. E., \& Chartier, K. (2006). Attrition in randomized controlled clinical trials: Methodological issues in psychopharmacology. Biological Psychiatry, 59, 1001-1005. https://doi.org/10.1016/j. biopsych.2005.10.020

Macafee, D., West, J., Scholefield, J. H., \& Whynes, D. K. (2009). Hospital costs of colorectal cancer care. Clinical Medicine: Oncology, 3, 2737. https://doi.org/10.4137/CMO.S2362

MacMillan Cancer Support. (2015). Hidden at home: The social care needs of people with cancer. https://edin.ac/39HvTFg, Accessed July 20, 2020.

Maniadakis, N., Fragoulakis, V., Pectasides, D., \& Fountzilas, G. (2009). Xelox versus folfox6 as an adjuvant treatment in colorectal cancer: an economic analysis. Current Medical Research and Opinion, 25, 797-805. https://doi.org/10.1185/03007990902719117

Mar, J., Errasti, J., Soto-Gordoa, M., Mar-Barrutia, G., Martinez-Llorente, J. M., Domínguez, S., García-Albás, J. J., \& Arrospide, A. (2017). The cost of colorectal cancer according to the tnm stage. Cirugía Española (English Edition), 95, 89-96. https://doi.org/10.1016/j. cireng.2017.01.001

Matter-Walstra, K., Schwenkglenks, M., Betticher, D., von Moos, R., Dietrich, D., Baertschi, D., \& Koeberle, D. (2016). Bevacizumab continuation versus treatment holidays after first-line chemotherapy with bevacizumab in patients with metastatic colorectal cancer: A health economic analysis of a randomized phase 3 trial (sakk 41/06). Clinical Colorectal Cancer, 15, 314-320. https://doi.org/10.1016/j. clcc.2016.03.002

Michalopoulos, N., Theodoropoulos, G., Stamopoulos, P., Sergentanis, T., Memos, N., Tsamis, D., Flessas, I., Menenakos, E., Kontodimopoulos, N., \& Zografos, G. (2013). A cost-utility analysis of laparoscopic vs open colectomy of colorectal cancer in a public hospital of the greek national health system. Journal of BUON, 18, 86-97.

Murphy, J., Halloran, S., \& Gray, A. (2017). Cost-effectiveness of the faecal immunochemical test at a range of positivity thresholds compared with the guaiac faecal occult blood test in the NHS Bowel Cancer Screening Programme in England. British Medical Journal Open, 7, e017186. https://doi.org/10.1136/bmjopen-2017-017186

Ó Céilleachair, A., Costello, L., Finn, C., Timmons, A., Fitzpatrick, P., Kapur, K., Staines, A., \& Sharp, L. (2012). Inter-relationships between the economic and emotional consequences of colorectal cancer for patients and their families: a qualitative study. BMC Gastroenterology, 12, 62. https://doi.org/10.1186/1471-230X-12-62

Ó Céilleachair, A., Hanly, P., Skally, M., O'Leary, E., O’Neill, C., Fitzpatrick, P., Kapur, K., Staines, A., \& Sharp, L. (2017). Counting the cost of cancer: out-of-pocket payments made by colorectal cancer survivors. Supportive Care in Cancer, 25, 2733-2741. https://doi. org/10.1007/s00520-017-3683-y

Observational Health Data Sciences and Informatics. (2021). Observational medical outcomes partnership (omop) common data model. https://www.ohdsi.org/data-standardization/the-commondata-model/, Accessed March 23, 2021.

Pettersson, K., Carlsson, G., Holmberg, C., \& Sporrong, S. K. (2012). Cost identification of nordic fliri, nordic flox, xeliri and xelox in first-line treatment of advanced colorectal cancer in sweden-a clinical practice model approach. Acta Oncologica, 51, 840-848. https://doi. org/10.3109/0284186X.2012.713507 
Pil, L., Fobelets, M., Putman, K., Trybou, J., \& Annemans, L. (2016). Costeffectiveness and budget impact analysis of a population-based screening program for colorectal cancer. European Journal of Internal Medicine, 32, 72-78. https://doi.org/10.1016/j.ejim.2016.03.031

Pilgrim, H., Tappenden, P., Chilcott, J., Bending, M., Trueman, P., Shorthouse, A., \& Tappenden, J. (2009). The costs and benefits of bowel cancer service developments using discrete event simulation. Journal of the Operational Research Society, 60, 1305-1314. https://doi.org/10.1057/jors.2008.109

PRISMA. (2020). Preferred reporting items for systematic reviews and metaanalyses (prisma). http://prisma-statement.org/, Accessed July 20, 2020.

Rao, C., Smith, F., Martin, A., Dhadda, A., Stewart, A., Gollins, S., Collins, B., Athanasiou, T., \& Myint, A. S. (2018). A cost-effectiveness analysis of contact $x$-ray brachytherapy for the treatment of patients with rectal cancer following a partial response to chemoradiotherapy. Clinical Oncology, 30, 166-177. https://doi.org/10.1016/j. clon.2017.11.015

Rautenberg, T., Siebert, U., Arnold, D., Bennouna, J., Kubicka, S., Walzer, S., \& Ngoh, C. (2014). Economic outcomes of sequences which include monoclonal antibodies against vascular endothelial growth factor and/or epidermal growth factor receptor for the treatment of unresectable metastatic colorectal cancer. Journal of Medical Economics, 17, 99-110. https://doi.org/10.3111/13696 998.2013.864973

Robles-Zurita, J., Boyd, K. A., Briggs, A. H., Iveson, T., Kerr, R. S., Saunders, M. P., Cassidy, J., Hollander, N. H., Tabernero, J., Segelov, E., Glimelius, B., Harkin, A., Allan, K., McQueen, J., Pearson, S., Waterston, A., Medley, L., Wilson, C., Ellis, R., ... Paul, J. (2018). Scot: a comparison of costeffectiveness from a large randomised phase iii trial of two durations of adjuvant oxaliplatin combination chemotherapy for colorectal cancer. British Journal of Cancer, 119, 1332-1338. https://doi.org/10.1038/s41416-018-0319-z

Sobhani, I., Alzahouri, K., Ghout, I., Charles, D. J., \& Durand-Zaleski, I. (2011). Cost-effectiveness of mass screening for colorectal cancer: Choice of fecal occult blood test and screening strategy. Diseases of the Colon and Rectum, 54, 876-886. https://doi.org/10.1007/ DCR.0b013e31820fd2bc

Špacírová, Z., Epstein, D., García-Mochón, L., Rovira, J., de Labry Lima, A. O., \& Espín, J. (2020). A general framework for classifying costing methods for economic evaluation of health care. The European Journal of Health Economics, 1-14.

Tilson, L., Sharp, L., Usher, C., Walsh, C., S, W., O'Ceilleachair, A., Stuart, C., Mehigan, B., John Kennedy, M., Tappenden, P., Chilcott, J., Staines, A., Comber, H., \& Barry, M. (2012). Cost of care for colorectal cancer in ireland: A health care payer perspective. The European Journal of Health Economics, 13, 511-524. https://doi.org/10.1007/ s10198-011-0325-z

Tscheulin, D. K., \& Drevs, F. (2010). The relevance of unrelated costs internal and external to the healthcare sector to the outcome of a cost-comparison analysis of secondary prevention: the case of general colorectal cancer screening in the german population. The European Journal of Health Economics, 11, 141-150. https://doi. org/10.1007/s10198-009-0156-3

van der Meulen, M. P., Lansdorp-Vogelaar, I., Goede, S. L., Kuipers, E. J., Dekker, E., Stoker, J., \& van Ballegooijen, M. (2018). Colorectal cancer: cost-effectiveness of colonoscopy versus CT colonography screening with participation rates and costs. Radiology, 287, 901911. https://doi.org/10.1148/radiol.2017162359

Whyte, S., Chilcott, J., \& Halloran, S. (2012). Reappraisal of the options for colorectal cancer screening in england. Colorectal Disease, 14, e547-e561. https://doi.org/10.1111/j.1463-1318.2012.03014.x

Woollard, M. (2014). 3.1 administrative data: Problems and benefits. A perspective from the united kingdom1. Facing the Future: European Research Infrastructures for the Humanities and Social Sciences, 49.

York Health Economics Consortium. (2016). A glossary of health economic terms. https://yhec.co.uk/resources/glossary/, Accessed June 1, 2020.

How to cite this article: Lemmon, E., Hanna, C.R., Hall, P., \& Morris, E.J.A. (2021). Health economic studies of colorectal cancer and the contribution of administrative data: A systematic review. European Journal of Cancer Care, 30(5), e13477. https://doi.org/10.1111/ecc.13477 
APPENDIX

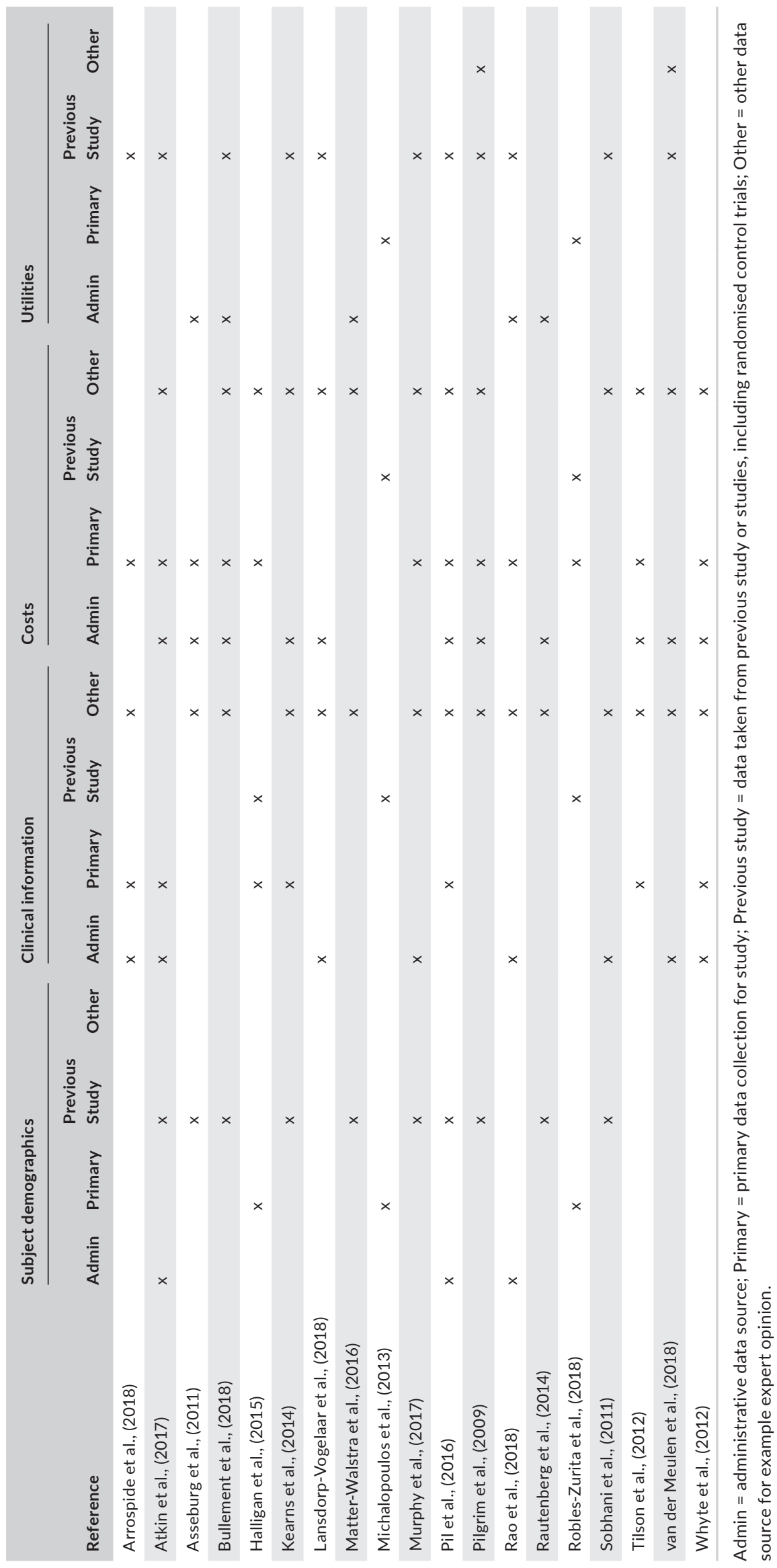




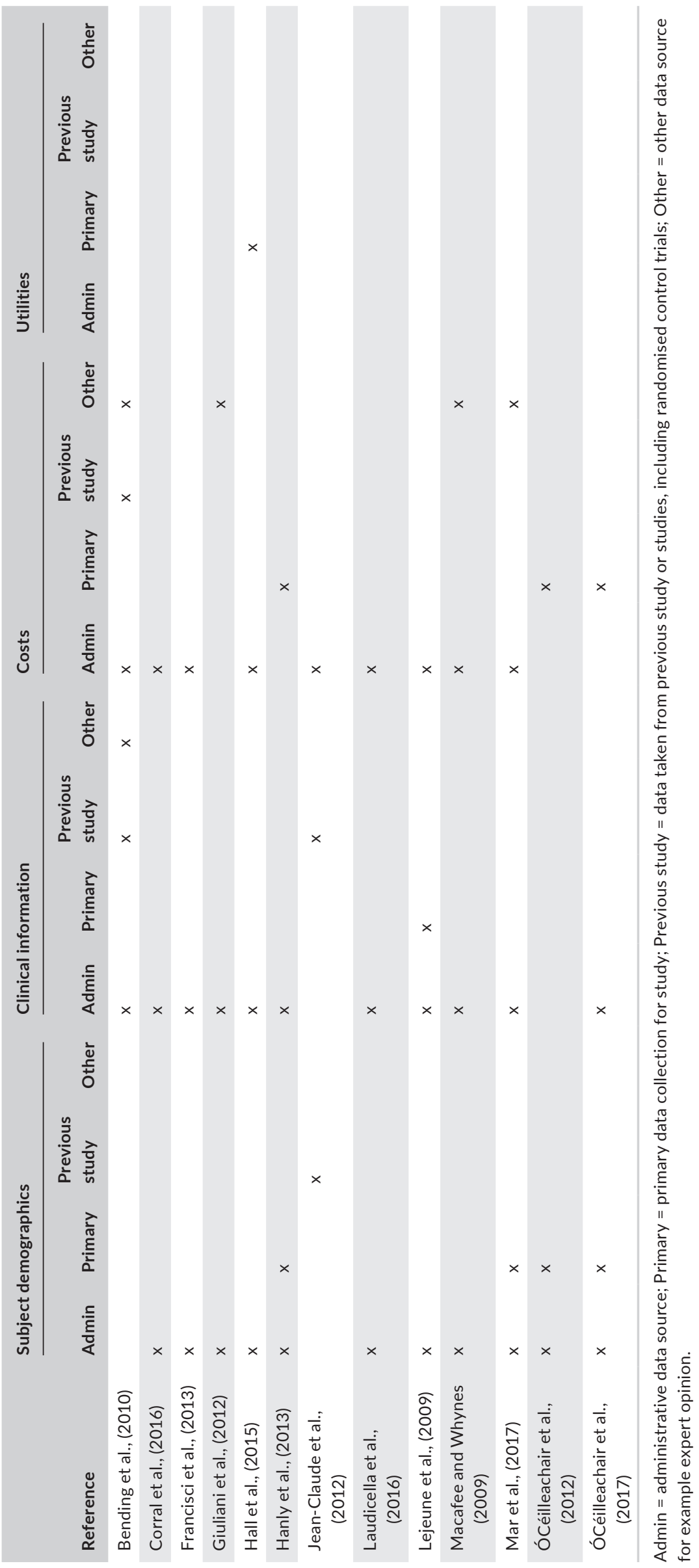




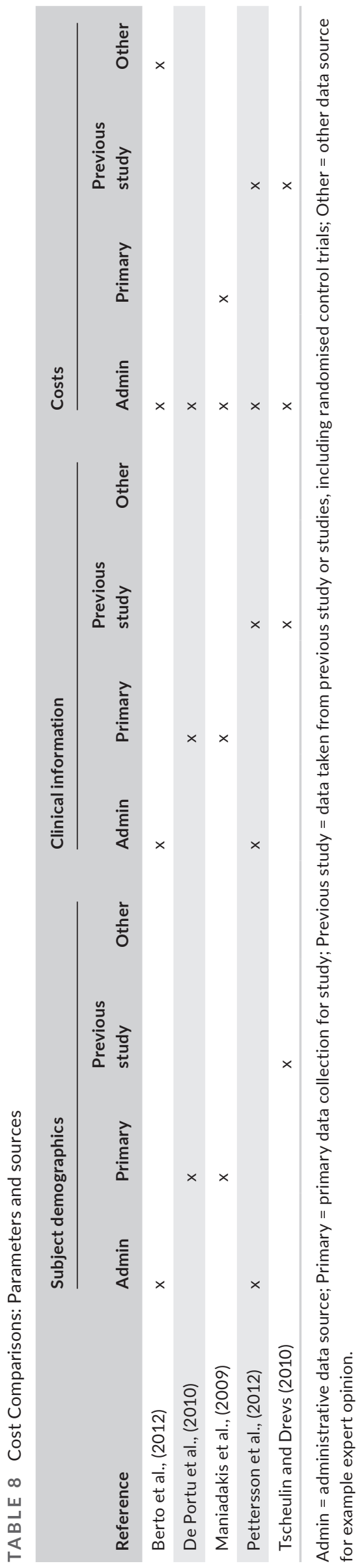

